James S. Krinsley

\title{
Understanding glycemic control in the critically ill: three domains are better than one
}

Received: 6 October 2010

Accepted: 8 November 2010

Published online: 6 January 2011

(C) Copyright jointly held by Springer and ESICM 2010

This editorial refers to the article available at:

doi:10.1007/s00134-010-2103-2.

\section{J. S. Krinsley ( $\square$ )}

Stamford Hospital, Stamford, USA

e-mail: JKrinsley@stamhealth.org

\section{J. S. Krinsley}

Columbia University College of Physicians

and Surgeons, New York, USA

Intensive insulin therapy targeting euglycemia burst into prominence 9 years ago with the publication of the singlecenter study from Leuven, Belgium, involving a cohort of 1,548 mechanically ventilated postoperative patients [1]. The prodigious influence of this landmark study is reflected by the Google Scholar website: 5,421 published manuscripts have cited this work in the 9 years since its publication [2]! Nevertheless, the early promise of this intervention, while corroborated in a large before and after study at my institution [3], and included in treatment guidelines by major organizations $[4,5]$, was not borne out in subsequent investigations. Two large multicenter studies $[6,7]$ were terminated prematurely by their safety boards for safety reasons-high rates of severe hypoglycemia-and protocol violations, and the Leuven group's trial in a medical population demonstrated only modest benefit [8]. Finally, the largest multicenter trial of allNICE-SUGAR - resulted in a small signal of harm in the intensively treated arm [9].
What went wrong? Each of the interventional trials focused on decreasing overall glycemic levels in the intensively treated arm, reporting mean morning glucose and mean glucose level as the main metrics. While the potential deleterious effect of severe hypoglycemia $(\mathrm{SH})$ was discounted in the sentinel investigation [1], later analysis of pooled data from the two Leuven studies demonstrated that SH was, indeed, independently associated with mortality [10]. Several large observational cohort studies confirmed this important finding [11-13]. Most recently, the role of glycemic variability, a parameter of glycemic control not considered in the design of any of the interventional trials, has been defined as an additional important contributor to the outcome of the critically ill-perhaps to an extent even greater than that contributed by reduction in the mean glucose level [14-17].

The current issue of Intensive Care Medicine features a large observational single-center study by Mackenzie and coinvestigators that sheds considerable light on this discussion [18]. The authors analyzed a total of 3,434 admissions over a 1-year period to four separate ICUs: surgical-liver; trauma-medical; cardiac surgical; neuroscience. Interestingly (especially in view of the fact that the study period ranged from July 2007 through June 2008), none of the units had in place a protocol for intensive insulin therapy. The study identified three discrete "domains" of glycemic control: measures of central tendency (four); measures of variability (eight); and a single metric of hypoglycemia, the minimum glucose value during ICU stay, and described for each their association with mortality.

This well-written article has a number of strengths. The use of blood gas analyzers avoided analytic issues relating to possible inaccuracies associated with the use of bedside glucometers. Four different patient populations were compared, increasing the generalizability of the study's conclusions. The size of the cohorts was large and the 
patients had a high number of glucose values [overall 15 (6-32) (median, interquartile range)]. The absence of information about the patients' diabetic status is an important limitation; recent literature has demonstrated that hyperglycemia has a different impact in critically ill diabetic and non-diabetic patients and, moreover, that glycemic variability may also have different implications in these two different groups [19-22]. Additionally, the authors did not have access to details of insulin therapy, so could not elucidate the impact of this therapy on modulating the relative influence of the three domains of glycemic control on mortality. Finally, the dataset did not include any severity of illness measure, precluding its use in a multivariable logistic regression model.

The association of mortality with the four measures of central tendency varied among the ICUs studied. Specifically, patients in the cardiac and surgical-liver unit experienced a "U"-shaped mortality curve, with better survival in the middle quintiles. Patients admitted to the trauma-medical unit sustained a linear relationship between increasing quintiles of this measure and mortality. Unexpectedly, patients admitted to the neurosciences unit demonstrated an "N"-shaped mortality curve, with higher mortality in the middle quintiles and lower mortality in the lowest and highest quintile. In contrast, the relationship between mortality and the eight metrics of variability as well as the single metric of minimum glucose was more consistent across patient populations. Increasing glycemic variability was consistently associated with increasing mortality and the domain reflecting hypoglycemia, minimum glucose level, was inversely related to mortality, with highest mortality among patients in the lowest quintile of minimum glucose.

Most significantly, Mackenzie et al. have demonstrated convincingly that each of the different domains of glycemic control affected mortality independently and that the effects of derangements in more than one domain were additive (see Table 3 in [18]). The odds ratios for mortality comparing patients in "low risk" vs. "high risk" groups for each of the three domains ranged from 2.0 to 2.5 when examining a single metric, 3.6-4.8 when analyzing two metrics together, and 6.0 when all three domains were combined.

The implications of these data are clear. The clinical trials using intensive insulin therapy to target euglycemia successfully achieved reductions in mean glucose level in the treated patients. However, this success came with a cost-high rates of hypoglycemia. Moreover, glycemic variability was not explicitly measured or managed in any completed trial. The Leuven investigators subsequently analyzed pool data from their two interventional trials and reported that severe hypoglycemia and increased glycemic variability each had an independent effect on mortality [10]. Notably, glycemic variability was increased by the intervention! It is possible, then, to describe the series of randomized trials that followed the initial surgical ICU trial [1] as a noble but misguided effort. The inability of these trials to demonstrate evidence of benefit can be attributed to their failure to successfully control and manage domains of glycemic control-the occurrence of hypoglycemia, and glycemic variability - that now have emerged as parameters at least as important as overall (mean or median) glycemic levels.

The compelling data presented by Mackenzie and coinvestigators in this issue of Intensive Care Medicine, in conclusion, are highly explanatory. Future trials of intensive glycemic management in critically ill patients must follow a different path than that taken by the completed trials. These new data confirm that in order to achieve optimum benefit for our patients, clinicians must manage all three domains of glycemic control-overall glucose levels (measures of central tendency), glycemic variability, and hypoglycemia. There is now a large body of literature that demonstrates that these goals cannot be achieved with intermittent monitoring of glucose levels, at intervals of 1-4 h. We eagerly await the implementation of new technology that allows continuous or nearcontinuous glycemic monitoring [23]. These tools will offer our community the best chance to simultaneously ameliorate all three domains of glucose control. The next chapter of the fascinating story of glycemic control in the critically ill is ready to begin!

\section{References}

1. Van den Berghe G, Wouters P, Weekers F, Verwaest C, Bruyninckx F, Schetz M, Vlasslaers D, Ferdinande P, Lauwers P, Bouillon R (2001) Intensive insulin therapy in critically ill patients. N Engl J Med 345:1359-1367

2. Google Scholar website (2010) http://scholar.google.com. Accessed 20 Sep 2010
3. Krinsley JS (2004) The effect of an intensive glucose management protocol on the mortality of critically ill adult patients. Mayo Clinic Proc 79:992-1000

4. American College of Endocrinology (2004) Position statement on inpatient diabetes and metabolic control. Endoc Prac 10:77-82
5. Dellinger RP, Carlet JM, Masur H, Gerlach H, Calandra T, Cohen J, GeaBanacloche J, Keh D, Marshall JC, Parker MM, Ramsay G, Zimmerman JL, Vincent JL, Levy MM, Surviving Sepsis Campaign Management Guidelines Committee (2004) Surviving Sepsis Campaign guidelines for management of severe sepsis and septic shock. Crit Care Med 32:858-873 
6. Preiser JC, Devos P, Ruiz-Santana S, Melot C, Annane D, Groeneveld J, Iapichino G, Leverve X, Nitenberg G, Singer P, Wernerman J, Joannidis M, Stecher A, Chioleiro R (2009) A prospective randomized multi-centre controlled trial on tight glucose control by intensive insulin therapy in adult intensive care units: the Glucontrol study. Intensive Care Med 35:1738-1748

7. Brunkhorst FM, Engel C, Bloos F, Meier-Hellmann A, Ragaller M, Weiler $\mathrm{N}$, Moerer O, GruendLing M, Oppert M, Grond S, Olthoff D, Jaschinski U, John S, Rossaint R, Welte T, Schaefer M, Kern P, Kuhnt E, Kiehntopf M, Hartog C, Natanson C, Loeffler M, Reinhart K (2008) Intensive insulin therapy and pentastarch resuscitation in severe sepsis. N Eng J Med 358:125-139

8. Van den Berghe G, Wilmer A, Hermans G, Meersseman W, Wouters PF, Milants I, Van Wijngaerden E, Bobbaers H, Bouillon R (2006) Intensive insulin therapy in the medical ICU. N Engl J Med 354:449-461

9. The NICE-SUGAR study investigators (2009) Intensive versus conventional insulin therapy in critically ill patients. N Engl J Med 360:1283-1297
10. Meyfroidt G, Keenan DM, Wang X, Wouters P, Veldhuis J, Van den Berghe G (2010) Dynamic characteristics of blood glucose time series during the course of critical illness: effects of intensive insulin therapy and relative association with mortality. Crit Care Med 38:1021-1029

11. Krinsley JS, Grover A (2007) Severe hypoglycemia in critically ill patients: risk factors and outcomes. Crit Care Med 35:2262-2267

12. Egi M, Bellomo R, Stachowski E, French C, Hart G, Taori G, Hegarty C, Bailey M (2010) Hypoglycemia and outcome in critical illness. Mayo Clinic Proc 85:217-224

13. Bagshaw S, Bellomo R, Jacka M, Egi M, Hart G, George C (2009) The impact of early hypoglycemia and glycemic variability on outcome in critical illness. Crit Care 13:R91

14. Egi M, Bellomo R, Stachowski E, French CJ, Hart G (2006) Variability of blood glucose monitoring and short term mortality of critically ill patients. Anesthesiology 105:244-252

15. Krinsley JS (2008) Glycemic variability: a strong, independent predictor of mortality in critically ill patients. Crit Care Med 36:3008-3013

16. Hermanides J, Vriesendorp T, Bosman R, Zandstra D, Hoekstra J, DeVries J (2010) Glucose variability is associated with intensive care unit mortality. Crit Care Med 38:838-842
17. Ali NA, O'Brien JA, Dungan K, Phillips G, Marsh CB, Lemeshow S, Connors AF, Preiser JC (2008) Glucose variability and mortality in patients with sepsis. Crit Care Med 36:2316-2321

18. Mackenzie I, Whitehouse T, Nightingale P (2011) The metrics of glycaemic control in critical care. Intensive Care Med. doi: 10.1007/s00134-010-2103-2

19. Krinsley JS (2006) Glycemic control, diabetic status and mortality in a heterogeneous population of critically ill patients before and during the era of tight glycemic control. Semin Thorac Cardiovasc Surg 18:317-325

20. Krinsley JS (2009) Glycemic variability in critically ill patients: the impact of diabetes. J Diab Sci Tech 3:1292-1301

21. Graham B, Keniston A, Gajic O, Trillo A, Cesar A, Medvedev S, Douglas I (2010) Diabetes mellitus does not adversely affect outcomes from a critical illness. Crit Care Med 38:16-24

22. Egi M, Bellomo R, Stachowski E, French CJ, Hart GK, Hegarty C, Bailey M (2008) Blood glucose concentration and outcome of critical illness: the impact of diabetes. Crit Care Med 36:2249-2255

23. Joseph J, Hipszer B, Mraovic B, Chervoneva I, Joseph M, Grunwald Z (2009) Clinical need for continuous glucose monitoring in the hospital. J Diab Sci Tech 3:1309-1318 